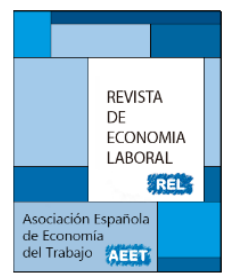

\title{
DINÁMICA DEL MERCADO LABORAL POR SEXO EN VENEZUELA: 1997-2012. ANÁLISIS DESCRIPTIVO ${ }^{1}$
}

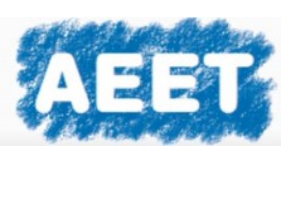

\author{
Mayra Montilla ${ }^{2}$ \\ Banco Central de Venezuela
}

Aceptado Marzo 2016

\section{Resumen}

Este trabajo tiene como objetivo estimar, analizar y comparar el comportamiento de los flujos de entrada y salida, y las probabilidades de transición instantáneas entre inactividad, desocupación y ocupación de hombres y mujeres en edad de trabajar (personas de 15 años y más) en Venezuela desde el 2do semestre de 1997 hasta el 1er semestre del 2012. Se contribuye a esta literatura mediante la inclusión de los flujos de entrada y salida del sector formal y el sector informal, ya que en Venezuela cerca del 48\% de las personas ocupadas se clasifican en el sector informal. A tal efecto, se aplica la metodología empleada en Fernández y Montilla (2015) clasificando a la población por sexo. Este trabajo encuentra que las mujeres tienen mayor movilidad laboral que los hombres, mayor probabilidad de transitar hacia la inactividad y menor probabilidad de encontrar empleo.

Palabras clave: Flujos brutos, probabilidades de transición instantáneas, sexo, Venezuela. Clasificación JEL: J49, J60, J16.

\section{Abstract}

This paper aims to estimate, analyze and compare the behavior of flows in and out, and the chances of instant transition between inactivity, unemployment and occupation of men and women of working age (persons 15 years and over) in Venezuela from the 2nd half of 1997 to the 1st half of 2012. Contributing to this literature by including the flows into and out of the formal sector and the informal sector, since in Venezuela about $48 \%$ of employed persons are classified in the informal sector. For this purpose, the methodology is applied in Fernandez and Montilla (2015) classifying the population by sex. This study found that women have greater mobility than men, more likely to move towards inactivity and less likely to find employment.

Key words: worker gross flows, instantaneous transition probabilities, sex, Venezuela.

JEL Classification: J49, J60, J16.

\footnotetext{
${ }^{1}$ Las opiniones expresadas en este trabajo son responsabilidad exclusiva del autor y no comprometen los de la Directiva del Banco Central de Venezuela. Se agradece los comentarios del Dr. León Fernández Bujanda, Investigador de Economía Senior del Banco Central de Venezuela, en el desarrollo de este trabajo. 2 Analista económico de la Oficina de Investigaciones Económicas del Banco Central de Venezuela. mmontil1@bcv.org.ve. Av. Urdaneta Esq. Las Carmelitas, 1010 Caracas, Venezuela. Teléfono: + 582125369215.
}

(C) Revista de Economía Laboral 


\section{Introducción}

A nivel mundial va en aumento la participación de la mujer en el mercado laboral, es decir, se viene produciendo un cambio en el mercado laboral que genera una mayor presencia de las mujeres, ya sea para cubrir necesidades básicas, enfrentar crisis económicas o simplemente porque ha crecido su rol protagónico como jefas de hogar.

En Venezuela ya, desde los años setenta, era indudable el proceso de mayor presencia de las mujeres en las aulas y en los centros de trabajo. La crisis económica que se inició en los años ochenta, y nos ha acompañado por todos estos años, exigió de las mujeres el desempeño acelerado de nuevos roles tanto a nivel educativo y a nivel social, como a nivel laboral. Sin embargo, los indicadores de la fuerza de trabajo venezolana evidencian desigualdad entre ambos sexos, hombres y mujeres, que sitúan en posición de desventaja a las mujeres, cuyas tasas de inactividad y desocupación son más altas que la de los hombres. En el período 1997-2012, en promedio, la tasa de inactividad de las mujeres es $49 \%$ y la tasa de desocupación es $13 \%$, mientras que los hombres, en promedio, tienen tasa de inactividad de 19\% y tasa de desocupación de $11 \%$. Estos valores muestran que, a pesar de los decretos de inamovilidad laboral ${ }^{3}$ publicados en Gaceta Oficial desde el año 2002 hasta finales del 2005, para proteger y garantizar el empleo en Venezuela, siguen existiendo limitaciones para que las mujeres accedan y permanezcan insertas en el mercado de trabajo.

Para muchos fines de investigación este nivel de análisis, observando sólo la variación de los stocks, es suficiente, sin embargo, esta variación puede esconder información relevante respecto de la heterogeneidad en el mercado laboral venezolano por sexo, principalmente, la dinámica de la participación laboral femenina, lo que motiva a analizar la movilidad labora ${ }^{4}$ por sexo en Venezuela.

La movilidad laboral es calculada mediante la estimación de flujos brutos de trabajadores, que tiene sus cimientos en el seguimiento de

\footnotetext{
${ }^{3}$ La inamovilidad laboral fue decretada por el Ejecutivo Nacional mediante el Decreto $\mathrm{N}^{\circ}$ 1752 y publicada en la Gaceta Oficial Extraordinaria $N^{\circ} 5585$ del 28 de abril del 2002 , para proteger y garantizar el empleo. El objetivo fundamental es que ningún trabajador sea despedido sin tener una causa justificada, desmejorado o trasladado de su centro de trabajo (Bonilla et. al, 2005).

El beneficio de la inamovilidad laboral para la mujer durante el embarazo y hasta por un año después del parto, el programa de alimentación de los trabajadores (LPAT) y la Ley Orgánica de Protección al Niño y al Adolescente (LOPNA).

${ }^{4}$ Movilidad en los diferentes estados de la fuerza de trabajo.
} 
personas entre un período y el siguiente. En Venezuela, la investigación de flujos brutos de Fernández y Montilla (2015) aportó importantes resultados sobre la movilidad laboral para la población total entre 1998 y 2012. En dicho trabajo, a partir de la base de datos de personas obtenidas mediante información de la Encuesta de Hogares por Muestreo (EHPM)-INE 5 , la principal encuesta de hogares del mercado de trabajo en el país, se construyen 29 páneles enlazados semestralmente, que son la fuente de las estimaciones de los flujos. A su vez, se aplica la metodología propuesta por Frazis et al. (2005) para estimar los flujos para la población total y se aplica la metodología propuesta por Shimer (2012) para corregir las estimaciones anteriores por el sesgo de agregación temporal. Separan el empleo en el sector formal y el sector informal para analizar los flujos entre cuatro estados laborales (inactivo, desocupado, sector formal y sector informal). Además, descomponen la contribución de cada flujo en la variación cíclica de la tasa de desempleo, tasa de ocupación y tasa de informalidad ${ }^{6}$.

El documento Fernández y Montilla (2015) encontró que la variación en la tasa de desocupación se asocia, principalmente, con el flujo de la ocupación hacia la desocupación. En otras palabras, el grupo de desocupados se amplía durante las recesiones ${ }^{7}$ en mayor parte por trabajadores que salen de la ocupación. Curiosamente, los trabajadores del sector informal no contribuyen a este flujo, resultado que confirma una mayor flexibilidad en el trabajador del sector informal, y por lo tanto una mayor variabilidad en la transición del empleo para este grupo. La contribución de la transición del desempleo al empleo a la variación cíclica de la tasa de desempleo es modesta; sin embargo, va en aumento después de los decretos de inamovilidad laboral. La probabilidad de transición de desocupación hacia ocupación cae en casi 10 puntos porcentuales en la recesión 2009-2010, mientras que apenas cambia en las dos primeras recesiones, 1998-1999 y 2002-2003, respectivamente.

\footnotetext{
5 INE: Instituto Nacional de Estadística.

6 Fernández y Montilla (2015) usan la definición según PREALC - OIT (1976) de la población ocupada en el sector informal de la economía: Personas ocupadas como servicio doméstico, trabajadores por cuenta propia no profesionales (tales como vendedores, artesanos, conductores, pintores, carpinteros, buhoneros, etc.). También se incluyen en este sector a los patronos, empleados, obreros y trabajadores familiares que trabajan en empresas con menos de cinco personas ocupadas. Por Tasa de informalidad se entiende a la relación entre la población ocupada en el sector informal con respecto al total de la población ocupada.

7 Dos trimestres consecutivos en caída del PIB real, que transformados en semestres corresponden a: $2^{\text {do }}$ semestre $1998-2^{\text {do }}$ semestre $1999,1^{\text {er }}$ semestre $2002-2^{\text {do }}$ semestre 2003 y $1^{\mathrm{er}}$ semestre $2009-2^{\text {do }}$ semestre 2010.
} 
El siguiente trabajo es una aplicación de la metodología empleada en Fernández y Montilla (2015) clasificando a la población por sexo. En detalle, el presente trabajo tiene como objetivo estimar, analizar y comparar el comportamiento de los flujos de entrada y salida, y las probabilidades de transición instantáneas entre inactividad, desocupación y ocupación de hombres y mujeres en edad de trabajar (personas de 15 años y más) en Venezuela desde el $2^{\text {do }}$ semestre de 1997 hasta el $1^{\text {er }}$ semestre del 2012 . Se contribuye a esta literatura mediante la inclusión de los flujos de entrada y salida del sector formal y el sector informal, ya que en Venezuela cerca del $48 \%$ de las personas ocupadas se clasifican en el sector informal. A tal efecto, se usan los 29 páneles enlazados semestralmente obtenidos en Fernández y Montilla (2015), se aplica la metodología propuesta por Frazis et al. (2005) adaptada para estimar los flujos para ambos sexos por separado y luego se aplica la metodología propuesta por Shimer (2012) para corregir las estimaciones anteriores por el sesgo de agregación temporal.

Los principales resultados de esta investigación son los siguientes: Las mujeres tienen mayor movilidad laboral que los hombres. Ellas tienen mayor probabilidad de mantenerse inactivas, cuya tendencia concuerda con la mayor tasa de inactividad, y mayor probabilidad de transitar desde la ocupación y desocupación hacia la inactividad, mientras que los hombres tienen mayor probabilidad de encontrar empleo. Sin embargo, para ambos sexos, durante las recesiones es más difícil encontrar empleo.

Otro grupo de resultados revela que el sector formal es altamente regulado y protegido para las mujeres, en otras palabras, la contratación de las mujeres se ha vuelto más rígida. Ellas tienen mayor movilidad entre el sector informal y la inactividad. Para ambos sexos, en la última recesión observada, las probabilidades de transición entre el sector formal y el sector informal caen considerablemente, lo que sugiere que la destrucción del empleo puede haber disminuido, así como la creación de empleo en el sector formal, tal como se menciona en el trabajo de Fernández y Montilla (2015).

El presente trabajo está organizado de la siguiente manera: En la sección 2 se revisa literatura relacionada al tema de estudio. En la sección 3 se consideran los datos y las definiciones de los indicadores oficiales del mercado laboral usados en este trabajo. En la sección 4 se realiza un análisis descriptivo de los indicadores tradicionales de la fuerza de trabajo por sexo en Venezuela. En la sección 5 se describe la metodología para estimar la movilidad laboral por sexo en Venezuela. En 
la sección 6 se analizan los resultados obtenidos y en la sección 7 se concluye este trabajo.

\section{Revisión de la literatura}

Investigaciones sobre dinámica del mercado laboral por sexo, en particular, movilidad laboral femenina, se centran en identificar variables que influyen en la dinámica aplicando modelos logísticos binomiales y multinomiales, y en las probabilidades de transición de los estados de la fuerza de trabajo usando matrices de Markov.

En algunos países de América Latina, como por ejemplo, Bolivia, Colombia, Ecuador, Perú y Venezuela, Goñi Pacchioni (2013) analiza la dinámica de empleo en estos países ${ }^{8}$ con base a las tasas de transición usando cadenas de Markov. Este autor observa que las mujeres transitan con mayor probabilidad hacia la inactividad.

En Colombia, Lasso (2013) analiza las tasas de transición entre cuatro estados laborales (asalariado, no asalariado, desocupado e inactivo) a partir del modelo de dos y tres estados propuesto por Shimer (2012). Este autor observa que las mujeres tienen menor probabilidad que los hombres de conservar sus empleos, que la probabilidad de transitar desde el desempleo o la inactividad a un empleo asalariado es casi la mitad que los hombres y que la probabilidad de transitar desde un empleo no asalariado o el desempleo hacia la inactividad es casi el doble.

En Costa Rica, Zuñiga et. al (2014) analizan los factores que explican la dinámica de transición entre tres estados (empleo, desempleo e inactividad). Estos autores observan que las mujeres tienen menor probabilidad de transitar del desempleo al empleo y mayores posibilidades de terminar la búsqueda de trabajo y pasar a la inactividad.

Los resultados de estos autores son similares a los obtenidos en este trabajo, sin embargo, la metodología aquí presentada es diferente, se obtienen las probabilidades de transición sin la aplicación de modelos logísticos.

En Venezuela, son escasos los antecedentes sobre la dinámica del mercado laboral por sexo, lo que motiva a la realización de este trabajo.

\footnotetext{
8 Países Andinos.
} 


\subsection{Mercado laboral por sexo en Venezuela. Antecedentes}

En Venezuela, los primeros antecedentes en relación al mercado laboral por sexo se centran en la participación laboral femenina y se sitúan en el año 1945, donde aparecen las primeras normas de la participación de la mujer (Constitución de Venezuela, 19459). Se establece la elección de cargos públicos sin distinción de sexo y el derecho al sufragio para formar los consejos municipales. En 1947, se establece el derecho de las mujeres al sufragio nacional, reposo remunerado antes y después del parto y salario igual para trabajo igual, sin distinción de sexo (Constitución de Venezuela, 1947). En la actualidad, la Constitución de la República Bolivariana de Venezuela, aprobada el 30 de diciembre de 1999 durante la presidencia de Hugo Chávez, establece la Ley de Igualdad de Oportunidades para la Mujer, cuyo artículo 4 se refiere a la "igualdad de oportunidades de hombres y mujeres, a través de políticas, planes y programas, sobre las bases de un sistema integral de seguridad social donde se asuman los aspectos de salud, educación, alimentación, recreación, trabajo y estabilidad laboral".

Luego, los decretos de inamovilidad laboral publicados en Gaceta Oficial desde el año 2002 hasta finales del 2005, para proteger y garantizar el empleo en Venezuela, en particular, a las mujeres, se ha convertido en una de las bases de la nueva Ley Orgánica del Trabajo, de los Trabajadores y Trabajadoras las (Ley Orgánica del Trabajo y de los Trabajadores) que fue promulgada en el año 2012.

Estudios sobre los determinantes de la participación de la mujer en el mercado laboral venezolano son amplios y se enfocan en identificar variables que influyen en dicha participación, como por ejemplo, la edad, el nivel educativo, el estado civil, la fecundidad. Entre estos estudios esta la relación entre el nivel educativo de las mujeres y el acceso al mercado laboral analizada por Orlando y Zuñiga (2001). Estos autores demuestran que los hombres devengan remuneraciones un $20 \%$ superiores que las mujeres con escolaridad y experiencia similar y desempeñándose en el mismo sector y ocupación. Sin embargo, según Acevedo (2005) el nivel educativo de las mujeres no es limitación para acceder al mercado laboral venezolano. En su estudio destaca tres tendencias en las desigualdades de sexo en el trabajo en Venezuela: i) desigualdades en el acceso al empleo; ii) precariedad del trabajo de las mujeres; y iii) desigualdad en las cargas reproductivas.

\footnotetext{
${ }^{9}$ Para más detalles ver: $\underline{\text { http://venciclopedia.com/?title=Constituciones_de_Venezuela }}$
} 
Vale destacar que, a pesar del desarrollo de una legislación laboral, en los últimos años, que busca equidad entre personas de diferente sexo, aún existe mayor vulnerabilidad de la mujer en el mercado de trabajo.

\section{Datos y Definiciones}

En este trabajo se utilizan los datos de la Encuesta de Hogares Por Muestreo (EHPM). Esta encuesta es la fuente de numerosas estadísticas correspondientes a la fuerza de trabajo o estado laboral, información demográfica detallada y características del empleo. Desde 1967 el Instituto Nacional de Estadísticas (INE) es el organismo ejecutor de la EHPM, aunque el presente documento se centra en el período 19972012. El diseño de la muestra de la EHPM es del tipo probabilístico estratificado bifásico ${ }^{10}$, cuyo tamaño ha variado en todo el período de interés. Antes del año 2001, el número de hogares seleccionados fue de alrededor de 16.000, mientras que quedó ampliado a cerca de 37.000 después de ese año ${ }^{11}$. La muestra está diseñada para producir estimaciones por Estados de características de la fuerza laboral, lo que significa que las familias de los 24 estados están en la encuesta. La frecuencia de la EHPM es semestral y cada hogar se entrevista una vez a lo largo de este período. El conjunto de datos del trabajo se compone de todas las personas cuya edad es de, al menos, 14 años en el período de la entrevista correspondiente.

Las definiciones de los indicadores utilizados en este trabajo son los siguientes:

Población Económicamente Activa: Personas de 15 años y más, con disposición y disponibilidad para trabajar en el periodo de referencia, que es la semana anterior al día de la entrevista. En cuanto a la Tasa de Actividad, se refiere al porcentaje de la población económicamente activa con respecto a la población de 15 años y más.

10 En el muestreo probabilístico cada elemento de la muestra puede tener una probabilidad distinta de ser seleccionado. Una muestra es estratificada cuando la población es previamente dividida en grupos de tal modo que cada elemento de la misma quede en uno y sólo uno de los grupos.

11 Entre el $1^{\text {er }}$ semestre del 2001 y el $1^{\text {er }}$ semestre del 2002 el INE realizó cambios metodológicos y de codificación en la EHPM: cambio de codificación de las variables control y entidad (Vargas antes del $1^{\mathrm{er}}$ semestre del 2001 era el Municipio Vargas del Distrito Capital). Los indicadores por Estados publicados por el INE en www.ine.gov.ve están disponibles a partir del $2^{\text {do }}$ semestre del 2001. 
Población Económicamente Inactiva: Personas de 15 años y más, estudiantes, amas de casa, rentistas, pensionistas, jubilados y trabajadores familiares que trabajan menos de 15 horas a la semana. Para pertenecer a este grupo estas personas deben además cumplir con la condición de no haber hecho gestiones para conseguir empleo en el periodo de referencia. La Tasa de Inactividad es el porcentaje de la población económicamente inactiva con respecto a la población de 15 años y más.

Población Ocupada: Personas de 15 años y más, quienes declararon que estaban trabajando o tenían un empleo durante la semana anterior al día de la entrevista. Por Tasa de Ocupación se entiende al porcentaje de la población ocupada con respecto a la población económicamente activa.

Población Desocupada: Personas de 15 años y más, quienes declararon que durante la semana anterior al día de la entrevista no estaban trabajando y estaban buscando trabajo con remuneración. Se incluyen aquellas personas que nunca han trabajado y buscan trabajo por primera vez. La Tasa de Desocupación es el porcentaje de la población desocupada con respecto a la población económicamente activa.

Población ocupada en el sector formal de la economía (Definición según PREALC - OIT ${ }^{12}$, 1976): Personas que trabajan en empresas de cinco personas o más, tanto del sector público como del sector privado. También se incluyen a los trabajadores por cuenta propia profesionales universitarios. Por Tasa de formalidad se entiende al porcentaje de la población ocupada en el sector formal con respecto al total de la población ocupada.

Población ocupada en el sector informal de la economía (Definición según PREALC - OIT, 1976): Personas ocupadas como servicio doméstico, trabajadores por cuenta propia no profesionales (tales como vendedores, artesanos, conductores, pintores, carpinteros, buhoneros, etc.). También se incluyen en este sector a los patronos, empleados, obreros y trabajadores familiares que trabajan en empresas con menos de cinco personas ocupadas. Por Tasa de informalidad se entiende a la relación entre la población ocupada en el sector informal con respecto al total de la población ocupada.

Se reproducen los indicadores oficiales del mercado de trabajo (tasa de actividad, tasa de inactividad, tasa de ocupación, tasa de desocupación, porcentaje de formalidad, porcentaje de informalidad) con

\footnotetext{
12 PREALC: Programa de Empleo para América Latina y el Caribe, OIT: Organización
} Internacional del Trabajo. 
los pesos de muestreo de las variables de: la población activa, inactiva, ocupada, desocupada, en el sector formal y en el sector informal, y las definiciones anteriores.

Con los datos de la EHPM se puede formar un conjunto de datos de panel para los fines del análisis. Los datos de panel son ampliamente utilizados en economía laboral. Según la definición del Bureau of Labor Statistics ${ }^{13}$, los datos de panel son aquellos en que las mismas unidades muestrales son observadas a través de varios períodos de tiempo.

El diseño de la muestra se basa en un esquema de rotación en el que una sexta parte de las viviendas en el período de la encuesta anterior se sale del juego en cada nuevo período de la encuesta. No hay identificadores individuales únicos en la encuesta, de donde, se debe crear un algoritmo para identificar a las viviendas coincidentes a través de los períodos consecutivos, luego los hogares dentro de las viviendas emparejadas, y, finalmente, las personas dentro de los hogares emparejados. La muestra pareada representa alrededor del $71 \%$ de la muestra de la EHPM. Para los detalles de la creación de los datos de panel ver Fernández y Montilla (2015).

Una importante propiedad de dichos datos es la posibilidad que proporcionan para estimar flujos brutos, es decir, transiciones a través del tiempo entre diferentes estados de una variable categórica.

En el caso de los flujos brutos de los estados de la fuerza de trabajo, a saber: Inactivo $(I)$, Desocupado $(D)$ u Ocupado $(O){ }^{14}$, son la transición de personas entre estado laboral de un período a otro consecutivo. De forma que, en un momento dado, cada estado laboral está conformado por flujos de personas que entran al respectivo estado y por flujos que salen de él.

\section{Indicadores tradicionales de la fuerza de trabajo por sexo en Venezuela. Análisis descriptivo}

En esta sección se analiza el mercado laboral venezolano por sexo, basado en términos de stocks. En el período 1997-2012 no ha sido tan significativo el aumento de la participación de las mujeres en el mercado de trabajo. En la Gráfica 1 se observa que pasa de $46 \%$ en el $2^{\text {do }}$ semestre de 1997 a $54 \%$ en el $1{ }^{\text {er }}$ semestre del 2004, para luego disminuir al 50\% hasta el 2012, lo que evidencia la moderada incorporación de la mujer al

\footnotetext{
${ }^{13}$ U.S. Department of Labor, Bureau of Labor Statistics. BLS Glossary.

${ }^{14} \mathrm{O}$ cualquier otra subdivisión o apertura de categorías que de ellas se pudieran obtener, como por ejemplo: inactivo, desocupado, formal e informal.
} 
mercado de trabajo, sin embargo, en la segunda recesión se nota un aumento moderado, comportamiento que puede estar asociado al fenómeno del trabajador adicional o añadido ${ }^{15}$ (CEPAL et. al, 2006). Si se compara la tasa de participación femenina con la masculina se observa que, aunque la masculina es cercana al 30\% mayor que la femenina, la participación de los hombres desciende 4 puntos porcentuales, puesto que pasa de $82 \%$ en el $2^{\text {do }}$ semestre de 1997 a $78 \%$ en el $1^{\text {er }}$ semestre del 2012. Estas disminuciones pueden ser explicadas por el incremento del número de jubilados de regímenes especiales del sector público (educación, salud, fuerzas militares y empresas públicas) y pensionistas que tienen menos de 64 años ${ }^{16}$.

\section{Gráfica 1: Tasa de la Población Económicamente Activa. Venezuela, 1997-2012}

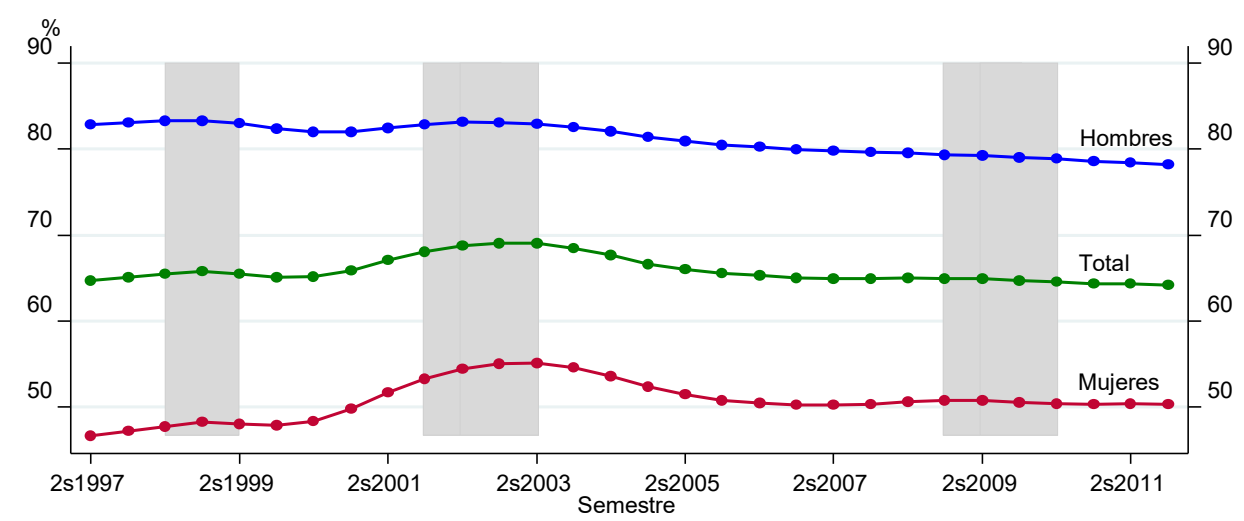

Fuente: Cálculo de los autores usando datos del BCV-INE.

Nota: Las regiones grises representan recesiones. Esta gráfica es obtenida aplicando media móvil a los datos originales.

15 El fenómeno del trabajador adicional se considera como estrategia de supervivencia y se produce cuando uno o más de los miembros del hogar queda en situación de desocupación y los inactivos pasan a formar parte de la oferta laboral (Zuñiga, 2007).

16 Para más detalles ver: http://www.ucv.ve/fileadmin/user_upload/auditoria_interna/Archivos/Material_de_Desc arga/Ley_del_Estatuto_Sobre_el_Regimen_de_Jubilaciones_y_Pensiones_de_los_Funcio narios_o_Empleados_de_la_Administracion_Publica_Nacional_-_38.426.pdf.

AVN- Emen (29 de diciembre de 2015). Venezuela aumentó a más de 3 millones la cifra de pensionados. El Mundo. 
Dentro de la Población Económicamente Activa (PEA), es importante evidenciar la evolución que han presentado sus componentes. La Gráfica 2 muestra una disminución de la tasa de ocupación femenina de $86 \%$ en el $2^{\text {do }}$ semestre de 1997 a $79 \%$ en el $1^{\text {er }}$ semestre del 2003 , para luego aumentar significativamente a $90 \%$ en el $1^{\mathrm{er}}$ semestre del 2012 . La tasa de ocupación de los hombres es cercana al 5\% mayor que las mujeres, lo cual podría estar relacionado con el tipo de actividades que generalmente realiza la población femenina, principalmente, en servicios comunales y sociales y en comercios, restaurantes y hoteles.

\section{Gráfica 2: Tasa de Ocupación. Venezuela, 1997-2012}

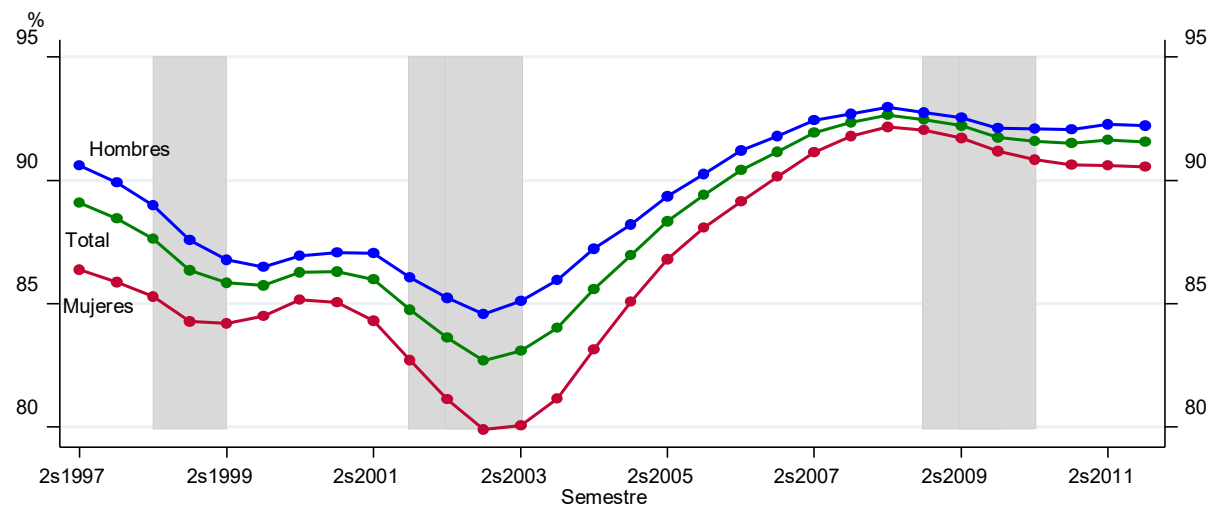

Fuente: Cálculo de los autores usando datos del BCV-INE

Nota: Las regiones grises representan recesiones. Esta gráfica es obtenida aplicando media móvil a los datos originales.

En cuanto a la tasa de desocupación, en la Gráfica 3 se observa que la mujer supera en 5\% a la de los hombres. En el período 1999-2001, la tasa de desocupación femenina ha mostrado momentos de importantes desaceleración. No obstante, la tendencia de disminución del desempleo femenino fue afectada por los sucesos de desestabilización ocurridos en el país entre el 2002 - 2003, pasando de $17 \%$ a $20 \%$, para comenzar a disminuir significativamente a partir del año 2004 pasando de $20 \%$ en el $1^{\text {er }}$ semestre del 2003 a $9 \%$ en el $1^{\text {er }}$ semestre del 2012, y lograr importantes resultados en la baja de este indicador mediante la generación de empleos para la inserción femenina. Durante el $2^{\text {do }}$ semestre del 2005 la tasa de desocupación femenina se ubicó en 14\%, lo que significa una reducción de 3\% en comparación al mismo período del año anterior que se ubicó en $17 \%$. 


\section{Gráfica 3: Tasa de Desocupación. Venezuela, 1997-2012}

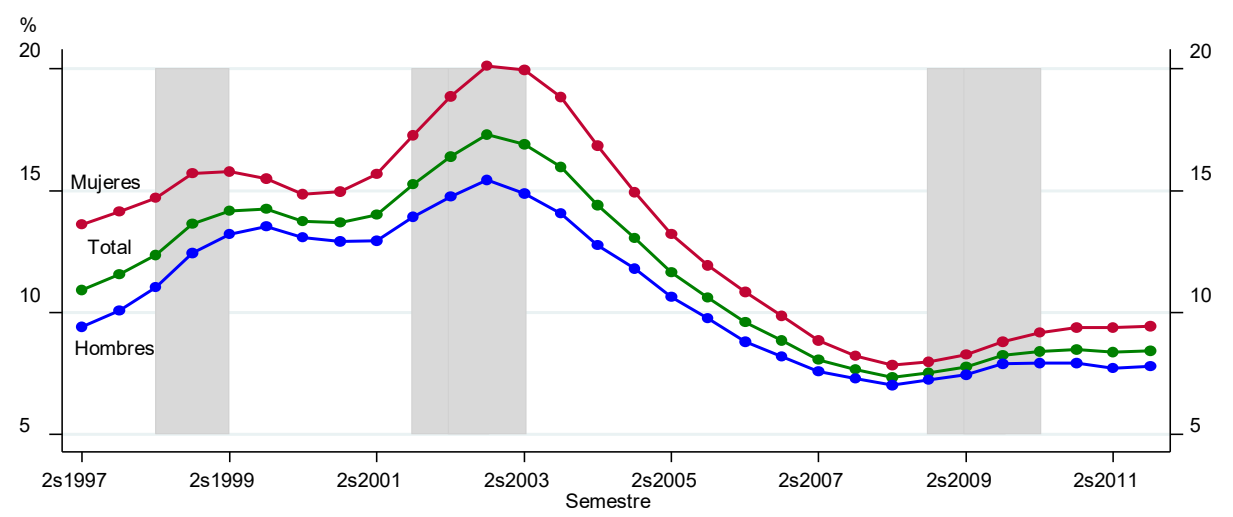

Fuente: Cálculo de los autores usando datos del BCV-INE.

Nota: Las regiones grises representan recesiones. Esta gráfica es obtenida aplicando media móvil a los datos originales

Sin embargo, el desempleo femenino sigue siendo más alto que el masculino (Iranzo y Richter, 2002). Para el $1^{\text {er }}$ semestre de 2007 la tasa de desempleo femenina era del 10\% en comparación con un $7 \%$ para los hombres. Aunque esta disparidad pareciera poca, la participación de la mujer en el mercado laboral venezolano es muy inferior al hombre, por ejemplo, de cada 11 personas que trabajan, 7 son hombres y 4 son mujeres.

Vale destacar que, a pesar del desarrollo de una legislación laboral en los últimos años que busca equidad entre personas de diferente sexo, aún existe mayor vulnerabilidad de la mujer en este mercado. El mayor desempleo femenino también está ligado a la poca contratación de mujeres adolescentes (Morillo, 2006).

En conclusión, la población femenina presenta una menor participación, sigue manteniendo menores niveles de ocupación y mayor desocupación en relación con los hombres.

Finalmente, considerando dentro del grupo de los ocupados a los ocupados clasificables ${ }^{17}$, el sector informal tiene una importante participación en el empleo total. En el período comprendido entre 19972012 , en promedio, la tasa de informalidad femenina es cercana al $47 \%$ y la masculina de $48 \%$, sólo un punto porcentual de diferencia. En la

17 Ocupados en el sector formal o en el sector informal. 
Gráfica 4 se muestra que el empleo informal afecta más a las mujeres entre el $1^{\mathrm{er}}$ semestre del $2001 \mathrm{y}$ el $2^{\text {do }}$ semestre del 2005 , con una tasa de $52 \%$ en el $1^{\text {er }}$ semestre del 2001 y $47 \%$ en el $2^{\text {do }}$ semestre del 2005 , sin embargo, a partir de este semestre es 5\% menor que la tasa de informalidad masculina, llegando a $39 \%$ en el $1^{\text {er }}$ semestre del 2012 . Se evidencia que después de la segunda recesión la tasa de informalidad femenina y masculina disminuye continuamente hasta la nueva recesión en el 2009, sin llegar a sus niveles anteriores. En cada recesión, la tasa de informalidad aumenta cerca de 3 puntos porcentuales, a excepción de la tercera recesión que no presenta cambios.

\section{Gráfica 4: Tasa de Informalidad. Venezuela, 1997-2012}

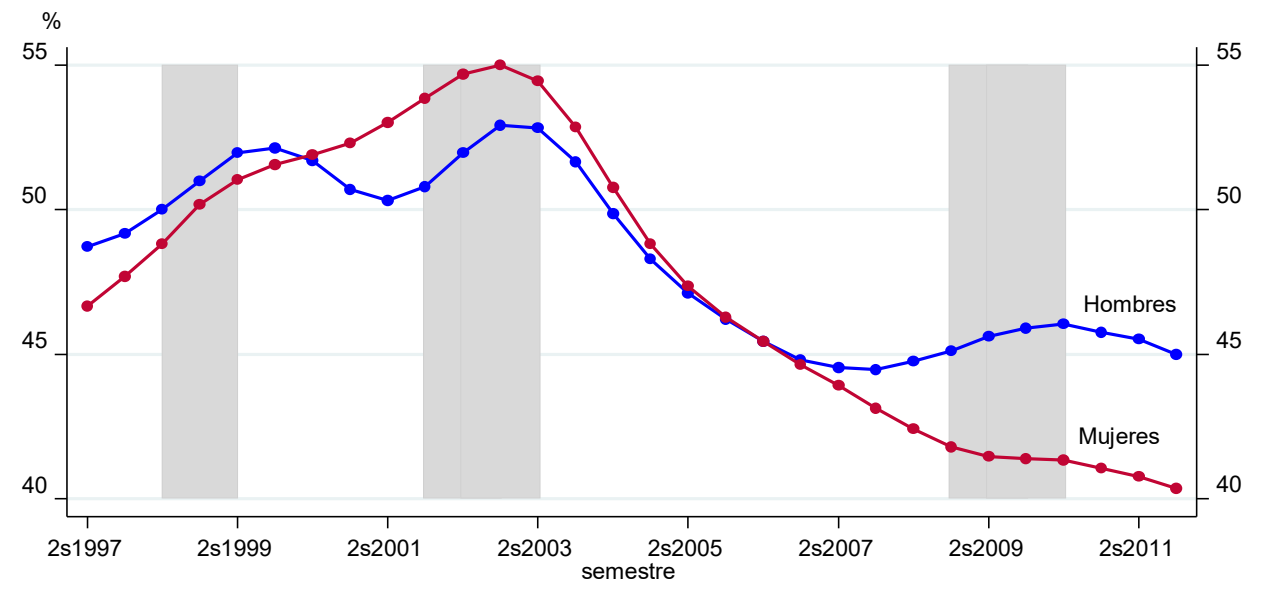

Fuente: Cálculo de los autores usando datos del BCV-INE

Nota: Las regiones grises representan recesiones. Esta gráfica es obtenida aplicando media móvil a los datos originales

La flexibilidad que proporciona el sector informal a las madres que trabajan conduce a una mayor incidencia de la informalidad en las mujeres que en los hombres. Los empleos formales y de alta productividad son escasos, de donde, las mujeres encuentran empleo en el sector informal.

\section{Metodología}

En esta sección se describe la metodología para estimar las transiciones de los estados laborales utilizada en Fernández y Montilla (2015) clasificando a la población por sexo. 


\subsection{Flujos brutos de los estados de la fuerza de trabajo en Venezuela por sexo}

Para entender el comportamiento del análisis tradicional del mercado laboral basado en términos de stocks, se aborda dicho análisis desde la perspectiva de los flujos brutos, en los que puede existir una considerable variabilidad en términos de transiciones entre los estados laborales, incluso en circunstancias en las que apenas se aprecian cambios en los stocks agregados netos.

Se aplica la metodología propuesta por Frazis et al. (2005) que consiste en recalcular o ajustar los flujos brutos de manera que coincidan con los stocks de entrada y salida. Es un método para corregir errores de márgenes ${ }^{18}$ denominado raking ${ }^{19}$.

En lo que sigue, se denotará Inactivos (I), Desocupados $(D)$, Ocupados $(O)$, Sector Formal $(F)$ y Sector Informal (Inf).

Como se mencionó anteriormente, en este trabajo se usan los datos de Fernández y Montilla (2015) para estructurar una tabla de flujos brutos entre los tres estados de la fuerza de trabajo: inactivo (I), desocupado $(D)$ y ocupado $(O)^{20}$, de semestre a semestre, como la que se muestra a continuación:

\footnotetext{
${ }^{18}$ Los errores de márgenes se refieren a que los cambios de stocks derivados de la suma de los flujos brutos no coinciden con los stocks oficiales.

19 Para más detalles ver Battaglia et al. (2003).

20 Se obtiene una tabla análoga cuando se consideran ocupados clasificables: formales $(F)$ e informales $(\operatorname{In} f)$.
} 
Cuadro 1: Estructura de la tabla básica de flujos brutos de los estados de la fuerza de trabajo: inactivos, desocupados y ocupados.

\begin{tabular}{|c|c|c|c|c|c|c|c|c|}
\hline & & & \multicolumn{5}{|c|}{ Semestre Actual } & \multirow{3}{*}{ Total fila } \\
\hline \multicolumn{3}{|c|}{ Estructura de Tabla Básica de Flujos Brutos } & \multicolumn{3}{|c|}{ Estado en la Fuerza Laboral } & \multicolumn{2}{|c|}{ Flujos Salientes } & \\
\hline & & & $I$ & $D$ & $O$ & Muertes & $\begin{array}{c}\text { Otros flujos } \\
\text { de salida }\end{array}$ & \\
\hline \multirow{5}{*}{ Semestre previo } & Estado en la & $I$ & $I I$ & $I a D$ & $\mathrm{IaO}$ & $m \_I$ & out_I & $I P$ \\
\hline & Fuerza Laboral & $D$ & $D a I$ & $D D$ & $\mathrm{DaO}$ & $m \_D$ & out_D & $D P$ \\
\hline & & $O$ & $O a I$ & $O a D$ & $O O$ & $m_{-} O$ & out_O & $O P$ \\
\hline & Flujos Entrantes & $\begin{array}{l}\text { Cumplen } 15 \text { años } \\
\text { Otros flujos de } \\
\text { entrada }\end{array}$ & $\begin{array}{c}I-14 \_15 \\
\text { in_I }\end{array}$ & $\begin{array}{c}D \_14 \_15 \\
\text { in_D }\end{array}$ & $\begin{array}{c}O_{-} 14+15 \\
\text { in_o }\end{array}$ & $\begin{array}{l}0 \\
0\end{array}$ & $\dot{0}$ & $\begin{array}{c}T_{-} 14 \_15 \\
P 1-P 0\end{array}$ \\
\hline & Total Columna & & $I A$ & $D A$ & $O A$ & $m$ & $P 0-P 1$ & Total \\
\hline
\end{tabular}

Fuente: Elaboración basada en Frazis et al. (2005). 
Los principales pasos en el procedimiento de estimación de flujos brutos, aplicados en el caso de Venezuela por sexo, son los siguientes:

1. Paso de ajuste de margen:

1.1. Construcción de los totales filas ( $I P, D P, O P)$ y columnas ( $I A, D A, O A)$ (totales oficiales) del semestre previo y semestre actual usando el peso ${ }^{21}$ del semestre respectivo.

1.2. Estimación del número de personas de 14 años en el semestre previo que pasan a 15 años en el semestre actual (I_14-15, D_14-15, O_14-15)

1.3. Estimación de muertes $\left(m_{-} I, m_{-} D, m_{-} O\right)$ tomando la población del semestre previo ${ }^{22}$ y multiplicándola por la tasa de muerte ${ }^{23}$ por sexo (de los indicadores INE, para personas de 15 años y más).

1.4. Estimación de los otros flujos de entrada y de salida: Sean: $P O=\left(I P+D P+O P+T_{-} 14-15\right)-\left(m_{-} I+m_{-} D+m_{-} O\right)$ $P 1=I A+D A+O A$

Si $P 1>P O$. se estiman in_I, in_D, in_ $O$ asignando $P 1-P O$ en proporción al semestre actual y los otros flujos de salida (outflow) son cero.

Si $P 1<P O$. se estiman out_I, out_D, out_O asignando $P O-P 1$ en proporción al semestre previo y los otros flujos de entrada (inflow) son cero.

\section{Paso de enlace:}

Construcción de los 9 flujos (II, IaD, IaO, DaI, DD, DaO, OaI, OaD, OO) usando el peso del semestre actual para personas coincidentes.

Este resultado no es enteramente consistente porque el estimado de las filas y columnas no coinciden con los totales filas y columnas oficiales, respectivamente. Para obtener consistencia se aplica el siguiente paso:

\section{Paso iteración:}

Los flujos entrantes y los flujos salientes se mantienen fijos, mientras las celdas de los estados en la fuerza laboral se ajustan iterativamente: Sea $k=I, D, O$

21 Número de unidades de la población representadas por la unidad muestral. Las estimaciones se hicieron ponderando cada observación por su respectivo peso.

22 Población total, inactivos, ocupados, desocupados.

${ }^{23}$ Para antes del año 2006 se utilizaron los anuarios de mortalidad- Ministerio de Salud.

(C) Revista de Economía Laboral 


\section{Ajuste Columna:}

Columna $k^{*}$ ajuste $k$

donde ajuste $k=\frac{k A-k_{-} 14 \_15-i n \_k}{E_{-} k A-k_{-} 14 \_15-i n \_k}$

\section{Ajuste Fila:}

Fila $k^{*}$ ajuste $k$

donde ajuste $k=\frac{k P-m_{-} k-o u t \_k}{E_{-} k P-m_{-} k-o u t_{-} k}$

La convergencia puede requerir un largo número de iteraciones (Battaglia et al. 2003).

La descripción de los flujos de los estados de la fuerza de trabajo (región sombreada del Cuadro 1) es la siguiente:

- De inactivo a desocupado (IaD): aspirantes (por ejemplo, estudiantes, amas de casa) y regresos.

- De inactivo a ocupado (IaO): contratación (en un instante es desocupado), dispuesto a aceptar un empleo.

- De desocupado a inactivo (DaI): retiro del mercado laboral, ya sea por desaliento del trabajador, jubilaciones, entre otros.

- De desocupado a ocupado $(\mathrm{DaO})$ : nuevas contrataciones, recontrataciones. También llamado flujo de creación de empleo.

- De ocupado a inactivo (Oal): retiro del mercado laboral, ya sea por desaliento del trabajador, jubilaciones, abandonos por salud, entre otros.

- De ocupado a desocupado $(O a D)$ : despidos, renuncias, fin de contratos, cierre de empresas. También llamado flujo de destrucción de empleo.

\subsection{Probabilidades de transición asociadas a los flujos brutos}

Para entender cómo funciona la dinámica de los flujos brutos de la fuerza de trabajo es útil conocer la probabilidad de transición con que una persona en un estado previo cambia de estado en el siguiente semestre. Dichas probabilidades se calculan de la siguiente manera:

$$
P_{t}^{k j}=\frac{k j}{k_{t-1}}
$$

donde $k, j=I, D, O, P_{t}^{k j}$ es la probabilidad de pasar del estado $k$ al estado $j$ durante el semestre actual t, $k_{\mathrm{t}-1}$ es el número total de personas 
en el estado $k$ en el semestre previo t-1 y kjes el número de personas que transitan del estado $k$ al estado $j$.

Las probabilidades de transición están relacionadas en el tiempo mediante la expresión matricial:

$$
\left(\begin{array}{c}
I_{t} \\
D_{t} \\
O_{t}
\end{array}\right)=\left(\begin{array}{ccc}
P_{t}^{I I} & P_{t}^{D I} & P_{t}^{O I} \\
P_{t}^{I D} & P_{t}^{D D} & P_{t}^{O D} \\
P_{t}^{I O} & P_{t}^{D O} & P_{t}^{O O}
\end{array}\right)\left(\begin{array}{c}
I_{t-1} \\
D_{t-1} \\
O_{t-1}
\end{array}\right)
$$

donde $\quad \sum_{k=I, D, O} P_{t}^{j k}=1$ para todo $j=I, D, O$ y $0<P_{t}^{k j}<1$.

Sea $\mathbf{P}_{\mathrm{t}}$ la matriz de las probabilidades semestrales de transición

$$
\mathbf{P}_{\mathrm{t}}=\left(\begin{array}{ccc}
P_{t}^{I I} & P_{t}^{I D} & P_{t}^{I O} \\
P_{t}^{D I} & P_{t}^{D D} & P_{t}^{D O} \\
P_{t}^{O I} & P_{t}^{O D} & P_{t}^{O O}
\end{array}\right)
$$

Como los autovalores de $\mathbf{P}_{\mathrm{t}}$ son reales, positivos y distintos ${ }^{24}$, se puede diagonalizar escribiendo $\mathbf{P}_{\mathrm{t}}=\mathbf{M}_{\mathrm{t}} \boldsymbol{\Lambda}_{\mathrm{t}} \mathbf{M}_{\mathrm{t}}^{-1}$ donde $\mathbf{M}_{\mathrm{t}}$ es la matriz cuyas columnas son los autovectores de $\mathbf{P}_{\mathrm{t}} \mathrm{y} \boldsymbol{\Lambda}_{\mathrm{t}}$ es la matriz diagonal con los autovalores de $\mathbf{P}_{\text {t. }}$.

\subsection{Probabilidades de transición en tiempo continuo}

Las estimaciones anteriormente mencionadas se corrigen por el sesgo de agregación temporal, que surge del hecho de que los flujos entre estados son un proceso continuo que se observa en períodos de tiempo discretos (semestre en este caso). En lo que sigue, se aplica la metodología de Shimer (2012). Sean $k, j=I, D, O$; se define $k j(\tau)$ como el número de personas que transita desde el estado $k$ en el semestre t hacia el estado $j$ en el momento $\tau$ donde $\tau \in[0,1)$; sea $k_{\mathrm{t}}$ el total de personas en el estado $k$ en el semestre t, dado por: $k_{\mathrm{t}}=\sum_{j=I, D, O} k j_{t}(\tau)$; se define $n_{t}^{k j}(\tau)$ para $k \neq j$ como la proporción de personas que estaban en el estado $k$ en el

${ }^{24}$ Calculados con el software estadístico Stata12. 
semestre t y en el estado $j$ en $t+\tau$, dado por: $n_{t}^{k j}(\tau)=\frac{k j_{t}(\tau)}{k_{t}}$ y satisface la ecuación diferencial ordinaria:

$$
\dot{n}_{t}^{k j}(\tau)=\sum_{l \neq j} n_{t}^{k l}(\tau) \lambda_{t}^{l j}-n_{t}^{k j}(\tau) \sum_{l \neq j} \lambda_{t}^{j l}
$$

donde $\lambda_{t}^{k j}$ es la tasa de ocurrencia (o tasa de transición instantánea) de un movimiento desde el estado $k$ al estado $j$, que se supone constante durante el período t a $t+\tau$.

Sea $A_{t}$ la matriz de transición instantánea ${ }^{25}$

$$
\mathbf{A}_{\mathrm{t}}=\left(\begin{array}{ccc}
-\lambda_{t}^{I D}-\lambda_{t}^{I O} & \lambda_{t}^{I D} & \lambda_{t}^{I O} \\
\lambda_{t}^{D I} & -\lambda_{t}^{D I}-\lambda_{t}^{D O} & \lambda_{t}^{D O} \\
\lambda_{t}^{O I} & \lambda_{t}^{O D} & -\lambda_{t}^{O I}-\lambda_{t}^{O D}
\end{array}\right)
$$

Usando la diagonalización de $\mathbf{P}_{\mathrm{t}}$ se puede escribir $\mathbf{A}_{\mathrm{t}}=\mathbf{M}_{\mathrm{t}} \log \Lambda_{\mathrm{t}} \mathbf{M}_{\mathrm{t}}{ }^{\text {. }}$ ${ }^{1}$ donde $\log \Lambda_{\mathrm{t}}$ es la matriz diagonal con los logaritmos de los autovalores de $\mathbf{P}_{\mathrm{t}}$. Una vez obtenidas estas tasas de transición, las probabilidades de transición en tiempo continuo se pueden calcular mediante la ecuación $\rho_{t}^{k j}=1-e^{-\lambda_{t}^{k j}}$.

\footnotetext{
${ }^{25} \mathrm{Si}$ se consideran los cuatro estados: inactivo, desocupado, formal e informal, $\mathrm{A}_{\mathrm{t}}$ se escribe de la forma:

$$
\left(\begin{array}{cccc}
-\lambda_{t}^{I D}-\lambda_{t}^{I F}-\lambda_{t}^{I n f} & \lambda_{t}^{I D} & \lambda_{t}^{I F} & \lambda_{t}^{I n f} \\
\lambda_{t}^{D I} & -\lambda_{t}^{D I}-\lambda_{t}^{D F}-\lambda_{t}^{D I n f} & \lambda_{t}^{D F} & \lambda_{t}^{D I n f} \\
\lambda_{t}^{F I} & \lambda_{t}^{F D} & -\lambda_{t}^{F I}-\lambda_{t}^{F D}-\lambda_{t}^{F I n f} & \lambda_{t}^{\text {Inf }} \\
\lambda_{t}^{\text {InfI }} & \lambda_{t}^{\text {InfD }} & \lambda_{t}^{\text {InfF }} & -\lambda_{t}^{\text {InfI }}-\lambda_{t}^{\text {InfD }}-\lambda_{t}^{\text {InfF }}
\end{array}\right)
$$
}




\section{Resultados}

\subsection{Flujos brutos de entrada y salida de inactivos, desocupados y ocupados por sexo en Venezuela}

Partiendo de los flujos brutos, medidos en base a la población en edad de trabajar de cada grupo, se nota un grado considerable de movilidad laboral por sexo en Venezuela: sobre el total de la población en edad de trabajar, en promedio, $23 \%$ de las mujeres experimentan alguna transición semestral de estado laboral. De donde, en promedio, 9\% transita a la ocupación, 5\% transita a la desocupación y 9\% transita a la inactividad, respectivamente. Mientras que los hombres, en promedio, 19\% experimenta alguna transición semestral de estado laboral. De donde, en promedio, $8 \%$ transita a la ocupación, 6\% transita a la desocupación y $4 \%$ transita a la inactividad, respectivamente.

En el Cuadro 2 se observa que la movilidad entre inactividad y ocupación es mayor en las mujeres. En promedio, 7\% de las mujeres ocupadas se mueve a la inactividad y el mismo porcentaje de las mujeres inactivas se mueve a la ocupación. Mientras que el flujo de los hombres entre estos dos estados es cercana al $3 \%$.

Cuadro 2: Promedio de los flujos brutos de entrada y salida de inactivos, desocupados y ocupados por sexo en Venezuela desde 1998 hasta el 2012.

\begin{tabular}{cccc}
\hline Flujo & Total & Hombres & Mujeres \\
\hline OO & 49,85 & 64,61 & 35,08 \\
OD & 3,41 & 4,68 & 2,14 \\
OI & 4,85 & 3,09 & 6,61 \\
DO & 3,56 & 4,82 & 2,30 \\
DD & 2,43 & 2,86 & 2,00 \\
DI & 1,83 & 1,19 & 2,46 \\
IO & 5,18 & 3,38 & 6,98 \\
ID & 1,98 & 1,35 & 2,61 \\
II & 26,71 & 13,99 & 39,43 \\
\hline
\end{tabular}

Fuente: Elaboración del autor.

Nota: Los flujos son medidos en términos de la población en edad de trabajar de cada grupo. Se aplica media móvil a los datos originales. 
La movilidad entre ocupación y desocupación es mayor en los hombres. En promedio, 5\% de los hombres ocupados se mueve a la desocupación y el mismo porcentaje de los hombres desocupados se mueve a la ocupación. Mientras que el flujo de las mujeres entre estos dos estados es cercana al $2 \%$.

Lo que evidencia que, los hombres desocupados tienden a conseguir trabajo, mientras que las mujeres se desalientan en la misma proporción que consiguen empleo. Una explicación a esta diferencia es la presencia de shocks idiosincráticos en el caso de las mujeres (Pries y Rogerson, 2009) y es consistente con la evidencia de los flujos analizados anteriormente, donde se vio que la mayor cantidad de las mujeres que dejan el trabajo lo hacen para irse a la inactividad. Si se considera este flujo como una decisión voluntaria, se puede suponer que las mujeres enfrentan más shocks idiosincráticos que las lleva a dejar la fuerza de trabajo, también la presencia de estos shocks puede explicar la mayor tasa de desempleo observada en las mujeres.

En promedio, 5\% de los hombres ocupados van a la desocupación cuando dejan de trabajar, mientras que 7\% de las mujeres ocupadas van a la inactividad. Bajo el supuesto de que el flujo desde ocupación hacia la desocupación corresponde a un abandono involuntario del trabajo y el flujo desde ocupación hacia la inactividad corresponde a un abandono voluntario del trabajo, entonces la mayoría de las mujeres dejan el empleo por razones personales, mientras que los hombres dejan el empleo forzadamente.

Se observa que el porcentaje del flujo desde inactividad hacia ocupación es mayor que desde inactividad hacia desocupación para ambos sexos, más notorio en las mujeres, lo que da a suponer que la incidencia del potencial de participación de las mujeres es la que ha estado imprimiendo el dinamismo de la fuerza de trabajo en Venezuela, son ellas quienes en mayor grado constituyen la reserva de factor trabajo afectando decisivamente las holguras relativas del mismo.

Los hombres disponen de una mayor probabilidad de mantenerse ocupados $(65 \%)$, mientras que las mujeres tienen una mayor probabilidad de permanecer inactivas (39\%). Las tendencias anteriores concuerdan con la mayor tasa de ocupación de los hombres y la mayor tasa de inactividad de las mujeres. Esto quiere decir que, mientras los hombres que pierden su empleo se mantienen procurando otro empleo, las mujeres están más propensas a formar parte de la población inactiva. Lo anterior refuerza la idea de que aún existe un comportamiento social donde son los hombres quienes asumen la responsabilidad de ser los proveedores del hogar, mientras las mujeres se encargan de las labores domésticas. 


\subsection{Flujos brutos de entrada y salida del sector formal y el sector informal por sexo en Venezuela}

Considerando los flujos de entrada y salida del sector formal e informal, en el Cuadro 3 se observa que, en promedio, 5\% de las mujeres en edad de trabajar fluyen al sector formal y $8 \%$ fluyen al sector informal. Mientras que $10 \%$ de los hombres en edad de trabajar fluyen al sector formal y $11 \%$ fluyen al sector informal. Lo que revela que el sector formal es altamente regulado y protegido para las mujeres.

Cuadro 3. Promedio de los flujos brutos de entrada y salida del sector formal y el sector informal por sexo en Venezuela desde 1998 hasta el 2012.

\begin{tabular}{cccc}
\hline Flujo & Total & Hombres & Mujeres \\
\hline F_F & 22,90 & 27,03 & 17,44 \\
Inf_Inf & 18,26 & 24,44 & 13,29 \\
F_D & 1,50 & 1,99 & 0,93 \\
Inf_D & 1,88 & 2,62 & 1,21 \\
F_I & 1,58 & 1,15 & 1,92 \\
Inf_I & 3,23 & 1,87 & 4,69 \\
D_F & 1,54 & 2,01 & 1,04 \\
D_Inf & 1,99 & 2,75 & 1,26 \\
I_F & 1,73 & 1,28 & 2,13 \\
I_Inf & 3,42 & 2,03 & 4,87 \\
F_Inf & 4,20 & 6,31 & 2,03 \\
Inf_F & 4,40 & 6,67 & 2,25 \\
\hline
\end{tabular}

Fuente: Elaboración del autor.

Nota: Los flujos son medidos en términos de la población en edad de trabajar de cada grupo. Se aplica media móvil a los datos originales.

En el Cuadro 3 se observa que la movilidad entre el sector informal y la inactividad es mayor en las mujeres. En promedio, 5\% de las mujeres en el sector informal se mueve a la inactividad y el mismo porcentaje de mujeres inactivas se mueve al sector informal. Mientras que el flujo de los hombres entre estos dos estados es cercana al $2 \%$. 
La movilidad entre el sector formal y el sector informal es mayor en los hombres. En promedio, 6\% de los hombres en el sector formal se mueve al sector informal y $7 \%$ que se encuentran en el sector informal se mueve al sector formal. Mientras que el flujo de las mujeres entre estos dos estados es cercana al $2 \%$.

Los hombres en el sector formal o en el sector informal van a la desocupación cuando dejan de trabajar, mientras que las mujeres van a la inactividad.

En promedio, 3\% de los hombres desocupados tienden a conseguir trabajo en el sector informal, mientras que $2 \%$ se mueven al sector formal.

Más hombres van desde el sector formal al sector informal que desde el sector formal a la desocupación, lo que puede reflejar la falta de un sistema de seguro de desempleo efectivo o la búsqueda de mejores oportunidades en mercados no regulados.

\subsection{Probabilidades de transición instantáneas entre inactividad, desocupación y ocupación por sexo en Venezuela}

El comportamiento dentro del mercado laboral, en función de la población en edad de trabajar, es diferente entre hombres y mujeres, no solo en los niveles presentados en la sección anterior, sino también en las probabilidades de transición.

Probabilidades de transición desde la ocupación.

En la Gráfica 5 se muestra que, para ambos sexos, el comportamiento cíclico de la serie de la probabilidad de transición desde la ocupación hacia la desocupación es similar: la probabilidad de perder el empleo y transitar a la desocupación aumenta durante las dos primeras recesiones. Sin embargo, en la recesión 2009-2010 no se observa un aumento en esta probabilidad, a pesar de que el empleo cae levemente durante este período. En cambio, en la expansión 2003-2009 la transición desde ocupación hacia la desocupación cae de manera permanente. 


\section{Gráfica 5: Probabilidades de Transición desde la Ocupación. Venezuela, 1s1998-1s2012}
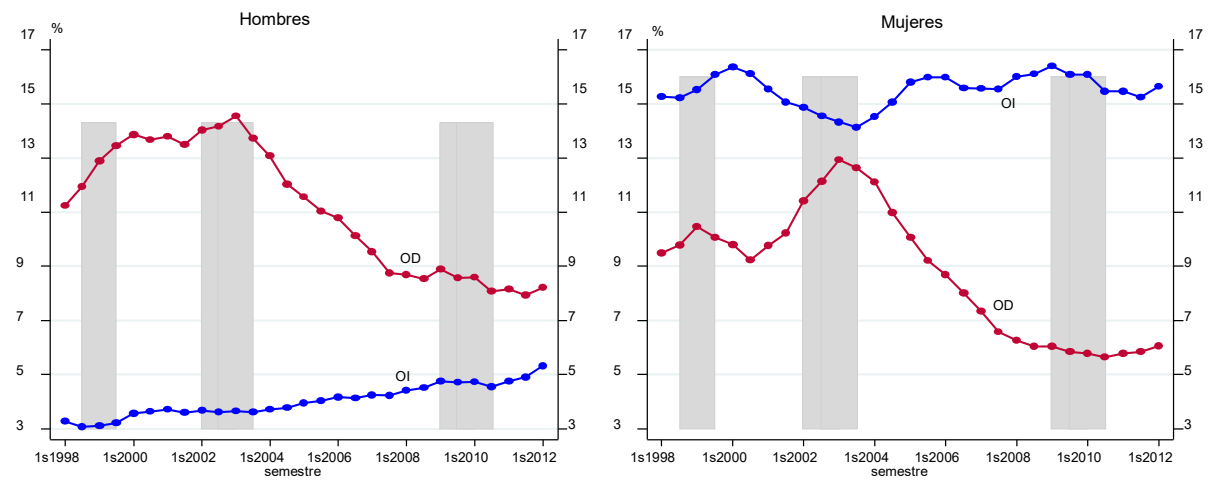

Fuente: Cálculo de los autores usando datos del BCV-INE

Nota: Las regiones grises representan recesiones. Esta gráfica es obtenida aplicando media móvil a los datos originales

Sube ligeramente la transición desde la ocupación hacia la inactividad a partir del 2005. Sin embargo, la probabilidad de transitar desde la ocupación hacia la inactividad es mucho mayor en las mujeres que en los hombres, en promedio, $12 \%$ mayor, de donde las mujeres ocupadas están más propensas a transitar hacia la inactividad. En cambio, los hombres ocupados son más propensos a ir a la desocupación que a la inactividad.

\section{Probabilidades de transición desde la desocupación.}

En la Gráfica 6 se observa que, para ambos sexos, la transición de desocupados hacia la ocupación cae en las recesiones. Es menos probable para los desocupados encontrar empleo. Esto es aún más evidente en la recesión 2009-2010, donde la probabilidad de transición de desocupados a ocupados presenta una caída de 10 y 5 puntos porcentuales para mujeres y hombres, respectivamente, y luego aumenta lentamente. En cambio, en la expansión 2003-2009, la transición de desocupados hacia el empleo aumenta de manera importante. 


\section{Gráfica 6: Probabilidades de Transición desde la Desocupación. Venezuela, 1s1998-1s2012.}

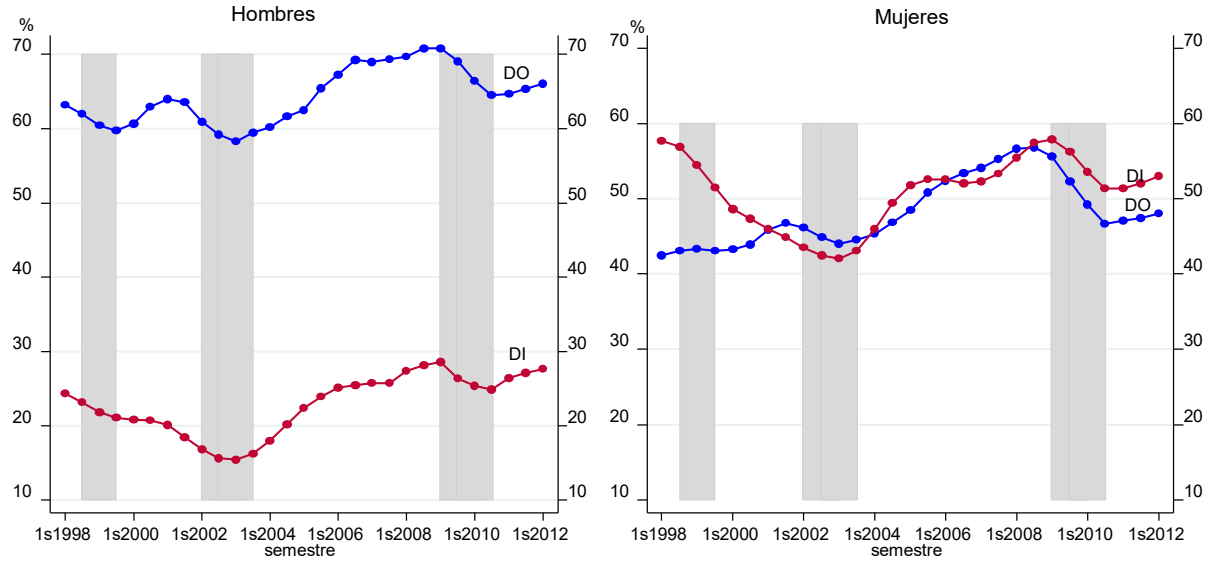

Fuente: Cálculo de los autores usando datos del BCV-INE

Nota: Las regiones grises representan recesiones. Esta gráfica es obtenida aplicando media móvil a los datos originales

En recesión, la probabilidad de transitar de desocupación hacia inactividad se reduce. Esto va contrario a la idea del trabajador desalentado o desanimado, correspondiente a aquel desempleado que en períodos en que se perciben reducidas oportunidades de emplearse abandona la fuerza laboral para formar parte de los inactivos.

Sube de manera permanente la transición desde la desocupación hacia la inactividad a partir del 2004, luego de haber caído desde 1998.

La probabilidad de transitar desde la desocupación hacia la inactividad es mucho mayor en mujeres que en los hombres, en promedio 25\% mayor, de donde las mujeres desocupadas están más propensas a transitar hacia la inactividad. En cambio, los hombres desocupados son más propensos a ir a la ocupación que a la inactividad. La evidencia sugiere que el mayor desempleo de las mujeres se relaciona con una menor probabilidad de encontrar empleo. 
Probabilidades de transición desde la inactividad.

En la Gráfica 7 se muestra que, para ambos sexos, en recesión, la probabilidad de transitar desde la inactividad hacia la desocupación aumenta moderadamente en los hombres, sin embargo, es importante evidenciar que desde mucho antes de la segunda recesión, la transición de mujeres inactivas a la desocupación aumenta 4 puntos porcentuales. Pasa de $9 \%$ en el $2^{\text {do }}$ semestre del 2000 a $13 \%$ en el $1^{\text {er }}$ semestre del 2002. Esto evidencia el efecto del trabajador adicional, que consiste en que en la parte baja del ciclo económico personas que usualmente no desean trabajar se incorporan a la fuerza de trabajo, por ejemplo, por miedo a que el principal sustento económico del hogar pierda el empleo o por necesidades básicas que satisfacer. En cambio, en la expansión 20032009, esta transición cae de manera importante. Por el contrario, la transición desde la inactividad hacia la ocupación disminuye en las recesiones.

\section{Gráfica 7: Probabilidad de Transición desde la Inactividad. Venezuela, 1s1998-1s2012.}

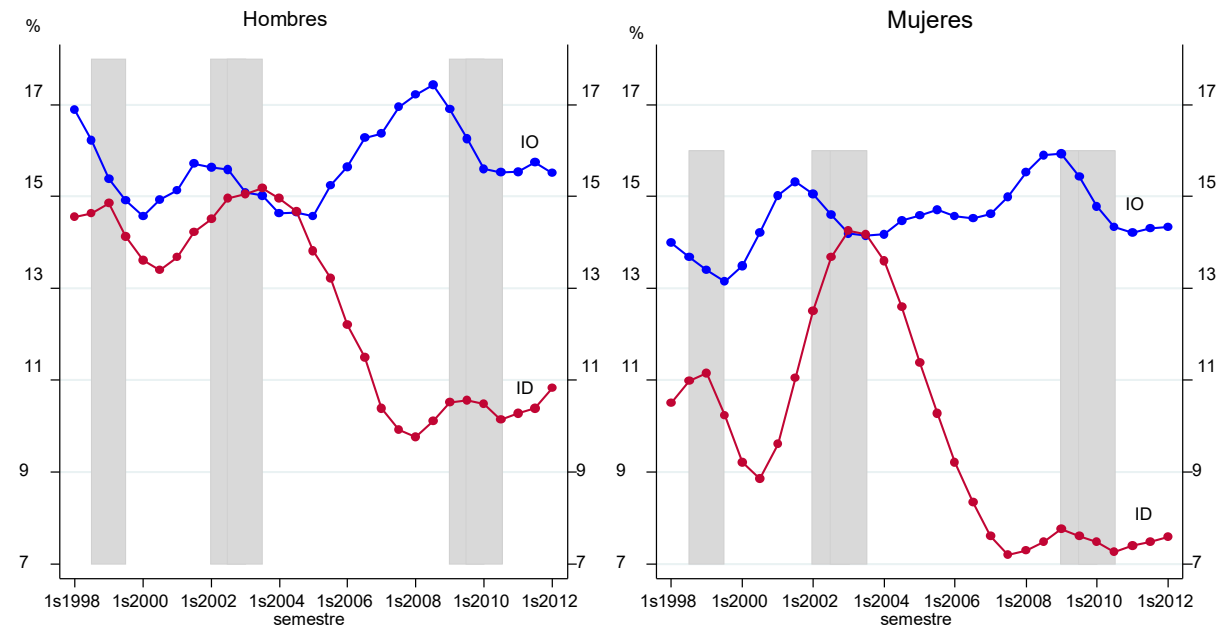

Fuente: Cálculo de los autores usando datos del BCV-INE.

Nota: Las regiones grises representan recesiones. Esta gráfica es obtenida aplicando media móvil a los datos originales. 
La probabilidad de transitar de inactividad hacia ocupación es mayor que la probabilidad de transitar de inactividad hacia desocupación por lo que una parte importante de los inactivos, a pesar de no estar buscando activamente un empleo, están dispuestos a aceptar un trabajo. Sin embargo, durante las crisis es más difícil encontrar un empleo.

La probabilidad de transitar de inactivo a ocupado es mayor en hombres que en mujeres, en promedio $2 \%$ mayor, de donde los hombres inactivos están más propensos a transitar hacia la ocupación, tienen mayor facilidad para volver a la fuerza de trabajo desde la inactividad.

\subsection{Probabilidades de transición instantáneas entre el sector formal y el sector informal por sexo en Venezuela}

Probabilidades de transición desde el sector formal.

En la Gráfica 8 se muestra que, para las mujeres, la probabilidad de transitar desde el sector formal hacia la desocupación aumenta durante las dos primeras recesiones, mientras que para los hombres apenas cambia. En cambio, para ambos sexos, en la expansión 2003-2009, la transición desde el sector formal hacia desocupación cae de manera permanente. Sin embargo, dicha transición tiende a disminuir después de las recesiones, a excepción de la mujeres donde en la recesión 20092010 tiende a aumentar levemente. 


\section{Gráfica 8: Probabilidades de transición desde el Sector Formal. Venezuela, 1s1998-1s2012.}
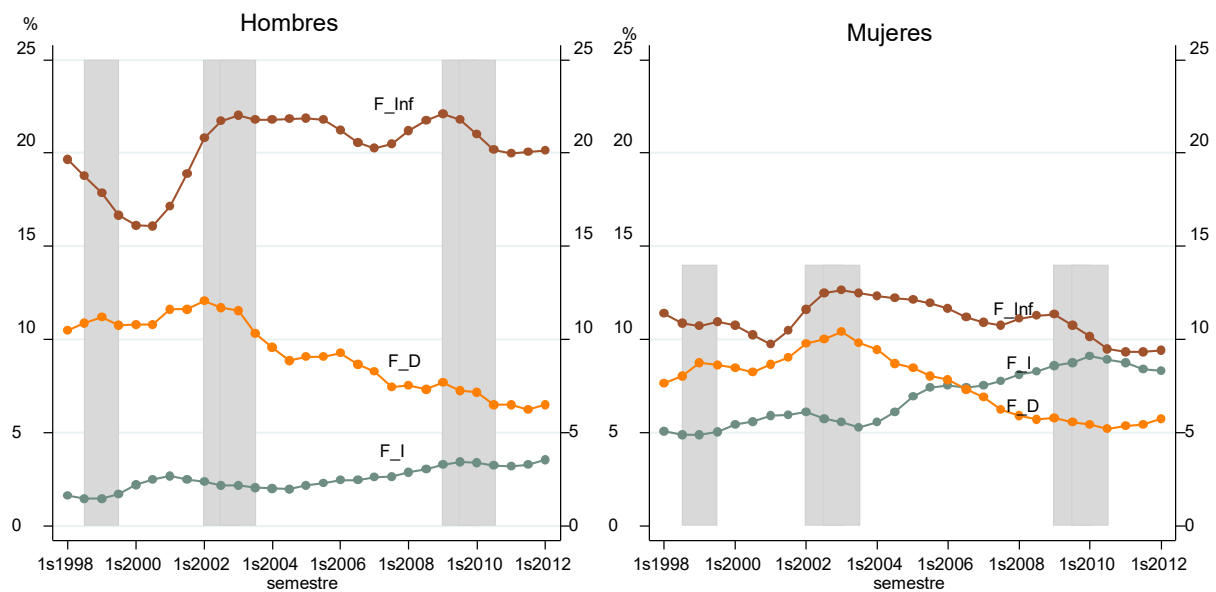

Fuente: Cálculo de los autores usando datos del BCV-INE.

Nota: Las regiones grises representan recesiones. Esta gráfica es obtenida aplicando media móvil a los datos originales.

Para los hombres, sube ligeramente la transición desde el sector formal hacia la inactividad a partir del 2005, sin embargo, para las mujeres aumenta significativamente esta transición, de $5 \%$ en el $1^{\text {er }}$ semestre del 2005 a $9 \%$ en el $2^{\text {do }}$ semestre del 2008.

Los hombres y las mujeres en el sector formal son más propensos $a$ ir al sector informal que a la inactividad.

La probabilidad de transitar desde el sector formal hacia el sector informal es mucho mayor en hombres que en las mujeres, en promedio $10 \%$ mayor, de donde los hombres en el sector formal están más propensos a transitar hacia el sector informal.

\section{Probabilidades de transición desde el sector informal.}

En la Gráfica 9 se muestra que, para ambos sexos, la probabilidad de transitar desde el sector informal hacia la desocupación aumenta durante las dos primeras recesiones. En cambio, en la expansión 20032009, la transición desde el sector informal hacia desocupación cae de manera permanente, de modo que en la última recesión no se observa un incremento de la misma. 


\section{Gráfica 9: Probabilidad de transición desde el Sector Informal. Venezuela, 1s1998-1s2012.}
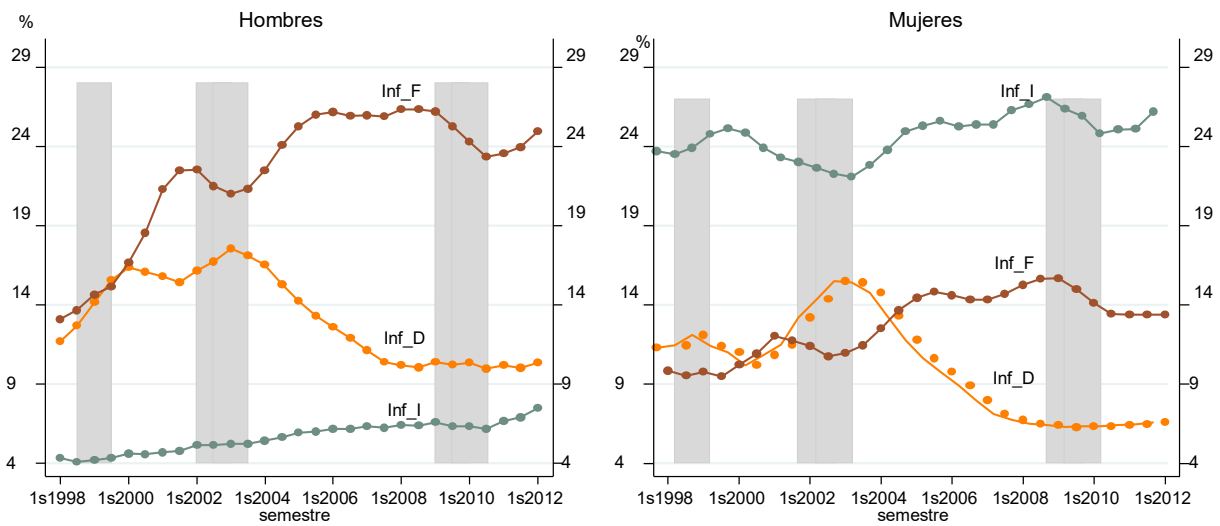

Fuente: Cálculo de los autores usando datos del BCV-INE.

Nota: Las regiones grises representan recesiones. Esta gráfica es obtenida aplicando media móvil a los datos originales.

Para hombres, sube ligeramente la transición desde el sector informal hacia la inactividad a partir del 2005, sin embargo, para las mujeres aumenta significativamente esta transición, de $23 \%$ en el $1^{\text {er }}$ semestre del 2005 a $30 \%$ en el $2^{\text {do }}$ semestre del 2008.

Los hombres en el sector informal son más propensos a ir a la desocupación o al sector formal que a la inactividad. Por el contrario, las mujeres en el sector informal son más propensas a ir a la inactividad que a la desocupación o al sector formal.

\section{Conclusiones}

En este trabajo se aplicó la metodología empleada en Fernández y Montilla (2015) clasificando a la población por sexo. En detalle, se estimaron, analizaron y compararon los flujos de entrada y salida y las probabilidades de transición instantáneas entre la inactividad, desocupación y ocupación de hombres y mujeres en edad de trabajar (personas de 15 años y más) en Venezuela desde el $2^{\text {do }}$ semestre de 1997 hasta el $1^{\mathrm{er}}$ semestre del 2012. Utilizando los datos de panel construidos en Fernández y Montilla (2015) se estimaron los flujos brutos por sexo aplicando la metodología de Frazis et al. (2005) para cada grupo por separado y se estimaron las probabilidades de transición instantáneas 
aplicando la metodología de Shimer (2012). Se contribuyó con la literatura en la inclusión de transiciones del sector formal e informal con el objetivo de analizar flujos de cuatro estados laborales.

Este documento encontró que:

- Los hombres, en edad de trabajar, tienen mayor probabilidad de mantenerse ocupados, mientras que las mujeres, en edad de trabajar, tienen mayor probabilidad de mantenerse inactivas. Las tendencias anteriores concuerdan con la mayor tasa de ocupación de los hombres y la mayor tasa de inactividad de las mujeres.

- Las mujeres tienen mayor movilidad laboral que los hombres. En promedio, 23\% de las mujeres, en edad de trabajar, experimentan alguna transición semestral de estado laboral, mientras que los hombres, en promedio, 19\% experimentan alguna transición semestral de estado laboral.

- La movilidad entre inactividad y ocupación es mayor en las mujeres.

- La movilidad entre desocupación y ocupación es mayor en los hombres.

- Los hombres desocupados tienden a conseguir trabajo, mientras que las mujeres se desalientan en la misma proporción que consiguen empleo. Esta diferencia puede ser explicada por la presencia de shocks idiosincráticos en el caso de las mujeres.

- La población femenina tiene mayores probabilidades de transitar desde la ocupación y desocupación hacia la inactividad y menor probabilidad de encontrar empleo, mientras que los hombres poseen mayores probabilidades de pasar desde la inactividad hacia la ocupación y desocupación, y mayor probabilidad de encontrar empleo.

- Para ambos sexos, la probabilidad de transitar desde la inactividad hacia la ocupación es mayor que la probabilidad de transitar desde la inactividad hacia la desocupación por lo que una parte importante de los inactivos, a pesar de no estar buscando activamente un empleo, están dispuestos a aceptar un trabajo.

- En recesiones se evidencia que: La probabilidad de perder el empleo y transitar a la desocupación aumenta durante las dos primeras recesiones, mientras que en la recesión 2009-2010 no se observa aumento. La probabilidad de encontrar empleo y de transitar desde la desocupación hacia la inactividad disminuye. La probabilidad de transitar desde la inactividad hacia la 
desocupación aumenta moderadamente en los hombres, sin embargo, se evidencia que desde mucho antes de la segunda recesión, la transición de mujeres inactivas a la desocupación aumenta significativamente. Este fenómeno es conocido, a veces, como el efecto del trabajador adicional.

- Otro grupo de conclusiones se refiere al flujo entre el sector formal y el sector informal. En promedio, 5\% de las mujeres en edad de trabajar fluyen al sector formal y $8 \%$ fluyen al sector informal. Mientras que, en promedio, $10 \%$ de los hombres en edad de trabajar fluyen al sector formal y $11 \%$ fluyen al sector informal. Lo que revela que el sector formal es altamente regulado y protegido para las mujeres.

- La movilidad entre el sector informal y la inactividad es mayor en las mujeres.

- La movilidad entre el sector informal y el sector formal es mayor en los hombres.

- Los hombres en el sector formal o en el sector informal van a la desocupación cuando dejan de trabajar, mientras que las mujeres van a la inactividad.

- Las mujeres y los hombres desocupados tienden a conseguir trabajo en el sector informal.

- Más hombres van desde el sector formal al sector informal que desde el sector formal a la desocupación, lo que puede reflejar la falta de un sistema de seguro de desempleo efectivo o la búsqueda de mejores oportunidades en mercados no regulados.

- Para ambos sexos, en la última recesión observada, las probabilidades de transición entre el sector formal y el sector informal caen considerablemente, lo que sugiere que la destrucción del empleo puede haber disminuido, así como la creación de empleo en el sector formal, tal como se menciona en el trabajo de Fernández y Montilla (2015).

Esta investigación permitió responder a preguntas como: ¿Bajo el enfoque estático y dinámico, el mercado laboral venezolano presenta desigualdad por sexo?, ¿las mujeres que acceden al mercado de trabajo permanecen insertas o tienen mayor movilidad?, ¿por sexo, es más flexible la ocupación en el sector formal o la ocupación en el sector informal?, ¿en recesión, es más difícil encontrar empleo para los hombres o las mujeres?

Se deja para futura investigación estimar el impacto de las legislaciones laborales, las transiciones demográficas y la creación de 
programas sociales sobre las transiciones de los estados laborales obtenidas en este trabajo.

\section{Referencias}

Acevedo, D. (2005). Desigualdades de sexo en el trabajo. Evolución y tendencias en la sociedad venezolana. Revista Venezolana de Estudios de la Mujer. 10(24).

Battaglia, M., Izrael, D., Hoaglin, D. y Frankel, M. (2003). Practical considerations in raking survey data. Tenth Biennial CDC \& ATSDR Symposium on Statistical Methods. Bethesda, MD. 1-37.

Bonilla, J. García, G. Rodríguez, M. (2005). Decretos de Inamovilidad Laboral en Venezuela (2002-2006). Revista sobre Relaciones Industriales y Laborales $\mathrm{N}^{\circ} 41.143-164$.

CEPAL, UNIFEM y otros (2006). Guía de Asistencia Técnica para la Producción y el uso de Indicadores de Sexo. Unidad Mujer y Desarrollo de la CEPAL. Santiago, Chile.

Fernández Bujanda León y Montilla Mayra (2015). Labor Flows in Venezuela: 1997-2012. Documento de Trabajo N 149. Banco Central de Venezuela.

Frazis, H. J., E. L. Robison, et al. (2005). Estimating gross flows consistent with stocks in the CPS. Monthly Labor Review. 128(9):3-9.

Goñi Pacchioni E., A. (2013). Andemic Informality: Assessing Labor Informality, Employment and Income Risk in the Andes. Washington, D.C. Inter-American Development Bank (IDB).

Iranzo, C. y Richter, J. (2002). El espacio femenino en el mundo del trabajo formal. Revista Venezolana de Gerencia. 7(20): 509 - 535. Lasso V. (2013). La dinámica del desempleo urbano en Colombia. En: Arango L. y Hamann F. (Eds). El mercado de trabajo en Colombia: hechos, tendencias e instituciones. Bogotá, D.C. Banco de la República. 
Morillo, M. (2006). Análisis del mercado laboral venezolano. FERMENTUM. 16(46): 501-552.

Orlando, M. y Zuñiga, G. (2001). Trabajo femenino y brecha de ingresos por género en Venezuela. Papeles de Población 27. México. 63-98.

PREALC-OIT (1976). El problema del empleo en América Latina: Situación, perspectivas y políticas. Santiago.

Pries, M., y Rogerson, R. (2009). Search frictions and labor market participation. European Economic Review. 53(5):568-587.

Shimer, R. (2012). Reassessing the Ins and Outs of Unemployment. Review of Economic Dynamics.15(2):127-148.

Zuñiga, G. (2007). Oferta Laboral Femenina. ¿Trabajadora adicional o trabajadora desalentada?, Cambio Demográfico en Venezuela, Oportunidades y Retos para las Políticas Públicas, III Encuentro Nacional de Demógrafos y Estudiosos de la Población, AVEPO, Caracas, Venezuela.

Zuñiga M., Garita J. y Vincenzi J. (2014). Análisis sobre la dinámica de transición y duración del desempleo en Costa Rica. Ciencias Económicas. 32(2): 39-64. 